\title{
Posterolateral approach for decompression with anterior and posterior fusion: a less invasive surgical technique for stabilization of the thoracic spine
}

\section{Case report}

\author{
Kaushik Das, M.D., Ahmed Rawanduzy, M.D., and William T. Couldwell, M.D., Ph.D. \\ Department of Neurosurgery, New York Medical College, Valhalla, New York
}

The goals of surgery in unstable thoracic fractures or tumors involving the thoracic spine are neural decompression, correction of deformity, and stabilization. Several different approaches can be used to achieve these goals. The anterior, combined anterior and posterior, and more recently thoracoscopic approaches are well described in the literature and are extremely effective in achieving the goals mentioned. However, they do carry with them significant morbidity and mortality rates in patients who are elderly, who have associated medical problems, or for whom there are contraindications to thoracic surgery. The authors believe the posterolateral approach is a viable alternative that is reasonably well tolerated in this select patient population. One can achieve circumferential neural decompression as well as anterior and posterior arthrodesis through a single incision. Most neurosurgeons are familiar with the anatomical landmarks and technical steps in the posterolateral approach, and the authors believe it is a less invasive method that can be especially useful and should not be overlooked in this subgroup of patients.

\section{Key Words * decompression * thoracic spine * posterolateral approach}

When decompression and fusion are needed in the management of disorders involving the thoracic spine, such as tumors or trauma, the anterior transcavitary approach or the combined anterior and posterior approaches carry with them significant rates of morbidity and mortality in patients who are elderly or have associated medical problems.[4,5] We believe that a posterolateral approach is a viable alternative and is reasonably well tolerated by this patient population. Using the posterolateral approach, one can achieve circumferential neural element decompression as well as good anterior and posterior arthrodesis.[1,8] Most neurosurgeons are quite familiar with the anatomical landmarks and technical steps in the posterolateral approach,[10] and we believe it is a less invasive method that can be useful especially in this subgroup of patients.

\section{SURGICAL TECHNIQUE}

Through a midline incision, we first perform a unilateral or bilateral laminectomy, followed by unilateral 
removal of the pedicle and facet and costotransversectomy. This posterolateral exposure provides good access to the posterior and lateral aspects of the spinal canal and neural elements. The posterolateral aspect of the vertebral body at this level is well visualized. If the patient is paraplegic or has radicular pain, the surgeon may decompress or sacrifice the exiting nerve root. The maneuver also provides additional space to access the vertebral body disease for proper decompression. Occasionally, removal of the medial portion of the contralateral pedicle may be helpful to relieve anterior compression safely and effectively, especially when there are retropulsed bone fragments. Once a satisfactory decompression is achieved, the surgeon can proceed with arthrodesis. We prefer to use pedicle screws and rods in patients who have minimum neural function. Gentle, gradual distraction allows significant deformity correction and facilitates placement of the interbody graft. Final hardware adjustment and tightening will place the graft under compression. In addition, posterior onlay graft is added to complete a posterior arthrodesis.

\section{ILLUSTRATIVE CASE}

Presentation. This 85-year-old man, who sustained a fall at home while working in the garden, presented 2 weeks later to an outside institution with progressive lower-extremity paresis. Initial magnetic resonance imaging studies performed at an outside institution revealed a compression fracture at T-6 with retropulsed fragments and spinal cord compression. He was managed conservatively with strict bed rest, and he gradually progressed to complete motor paralysis with some sensory sparing over a period of 2 weeks; repeated imaging showed progressively worsening kyphosis. He had also developed left lower lobe pneumonia. The patient was transferred to our institution for further management.

Examination. Neurological examination on arrival revealed complete paraplegia, urinary retention, and minimal retained sensation to deep pain below T-7. His most significant complaint was intractable radicular pain bilaterally, more severe on the left, which could be relieved only by large doses of narcotics. Repeated imaging showed a compression fracture of T- 6 with approximately $30 \%$ canal compromise, as well as a significant kyphotic deformity that had progressed despite strict bedrest (Fig. 1). Given his age and overall poor medical condition, a posterolateral decompression with anterior and posterior fusion was proposed with the goal of decreasing his pain, restoring alignment, and preserving the little remaining neurological function. 


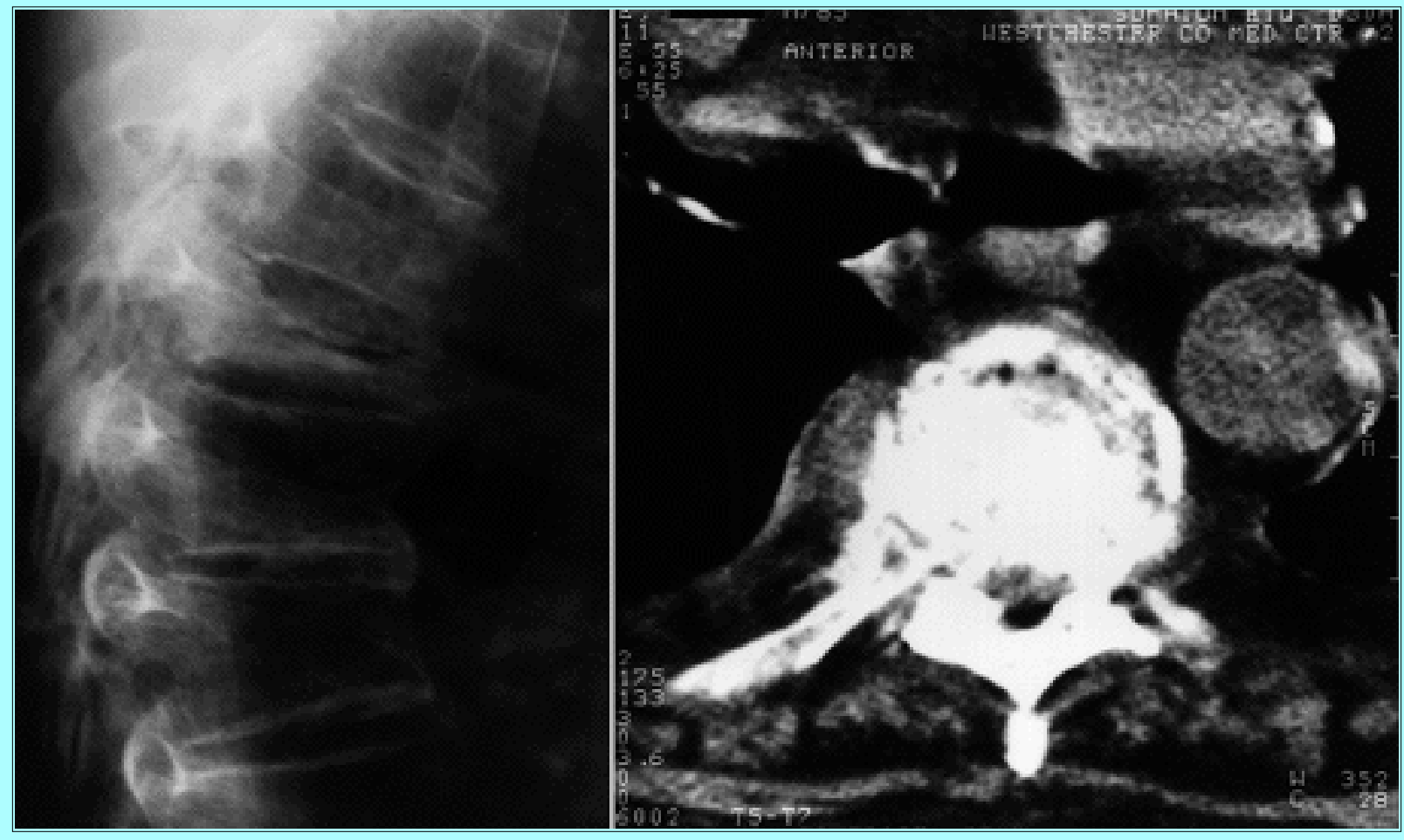

Fig. 1. Left: Preoperative plain x-ray film revealing a compression fracture of T-6 with severe kyphosis. Right: Computerized tomography scan demonstrating compromise of the spinal canal at the level of the fracture.

Operation. General anesthesia was induced in the patient and he was placed prone. Under fluoroscopic guidance and with neurophysiological monitoring and a midline incision was used to expose the region centered on T-6. A bilateral laminectomy and left costotransversectomy were completed. The fractured body of T-6 and the superior and inferior disc material (T5-6 and T6-7) were removed by using a high-speed drill and currettes from beneath the posterior longitudinal ligament. The cavity thus created facilitated disimpaction of the retropulsed bone fragments away from the spinal cord and allowed them to be removed piecemeal. To complete the circumferential decompression, the medial portion of the contralateral pedicle was also removed, facilitating the disimpaction of the remaining fragments on that side. Pedicle screws and rods were inserted and the T- 6 roots, which were severely damaged by the trauma, were sacrificed, bilaterally, with the hope of eliminating the patient's radicular pain. Careful distraction allowed correction of the deformity and positioning of an appropriately sized allograft for anterior arthrodesis. This was followed by final adjustment, placing the graft under compression. Posterior onlay bone allograft and autograft were added to complete posterior arthrodesis. No drains were used. Estimated blood loss was less than $150 \mathrm{ml}$. Postoperatively, the patient was mobilized rapidly and remained paraplegic but did experience a significant improvement in sensation below T-7. There were no postoperative complications and the radicular thoracic pain had disappeared. Postoperative imaging showed improvement of alignment and a well-decompressed canal (Fig. 2). 


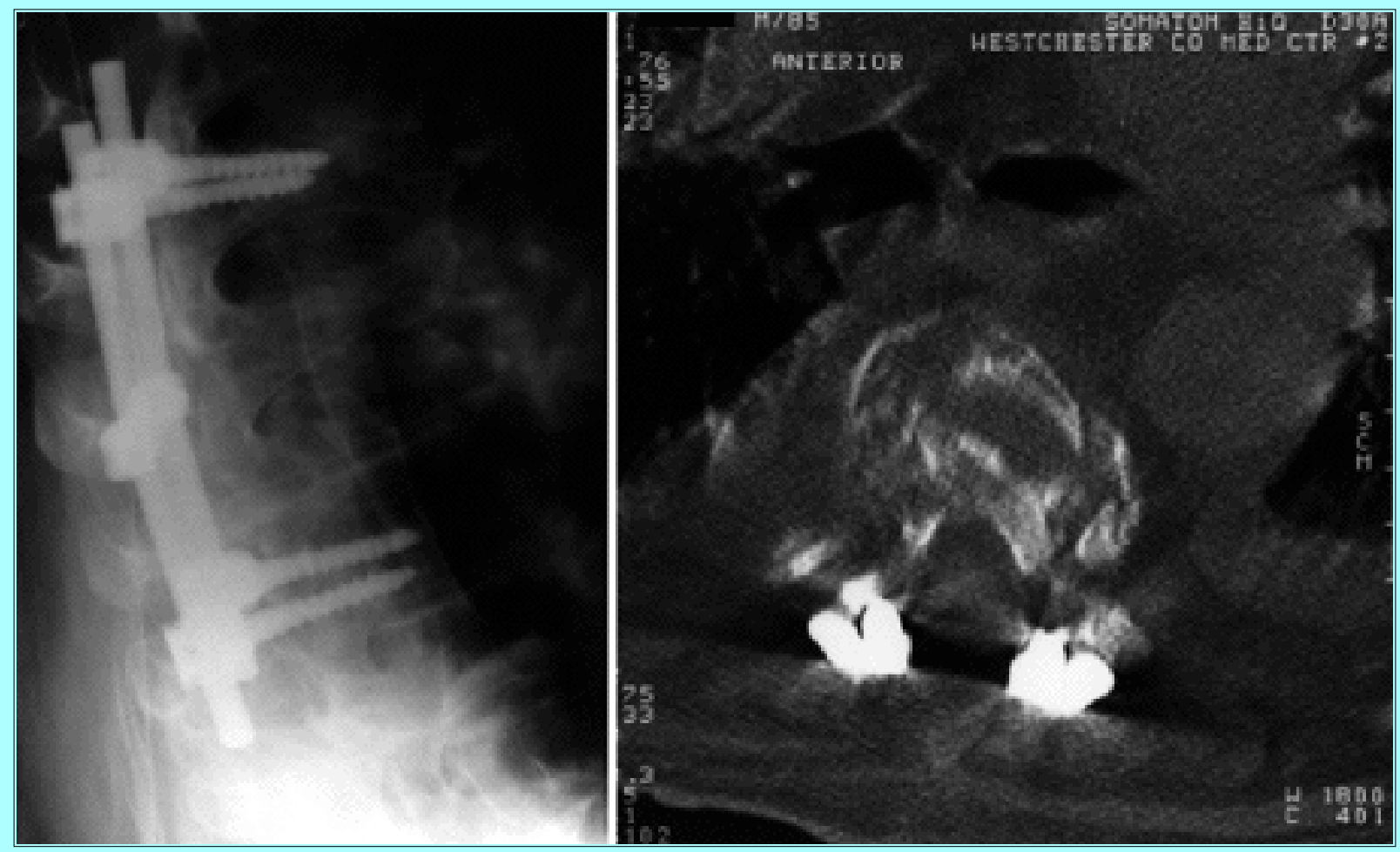

Fig. 2. Left: Postoperative plain x-ray film demonstrating significant improvement of kyphosis and transpedicular instrumentation. Right: Postoperative computerized tomography scan showing well-decompressed spinal canal.

\section{DISCUSSION}

The goals of surgery in unstable thoracic fractures or tumors involving the thoracic spine are neural decompression, correction of deformity, and stabilization.[6,8] These goals can be achieved by several different approaches. Anterior and combined anterior and posterior approaches to the thoracic spine are well described in the literature and are extremely effective in achieving the goals mentioned.[4,6] However, they do carry with them the risks associated with traversing the chest cavity, excessive blood loss, and in some cases, a protracted two-stage operation. More recently, thoracoscopic approaches[7,9] have also been introduced and used to perform anterior decompression and fusion. Although they are less invasive than standard open anterior approaches, the thoracoscopic approaches can still lead to significant blood loss and require multiple incisions and chest tubes at the conclusion of the operation.

As illustrated in the present case, the posterolateral approach[1,8] remains a viable, less invasive alternative in patients who are elderly, have multiple medical problems, or have contraindications for operations through the chest cavity. Through a single incision the approach exposes the neural elements for decompression and provides sufficient room for both anterior and posterior arthrodesis. This can be accomplished with relatively little blood loss in a single-stage operation, eliminating the need for drains or chest tubes that are required in transthoracic as well in thoracoscopic approaches. This familiar technique is flexible and can be tailored to the type of disease present. Endoscopic instruments can easily be used as an adjunct if the surgeon prefers. Stabilization with transpedicular screws and rods allows the correction of the kyphotic deformity and can be used to place the anterior graft under compression as well. This approach provides a short-segment posterior fixation, requiring less exposure and causing less blood loss than a longer rod and sublaminar hook construct. Although the techniques described in this report are well known, $[2,3]$ we believe that the combination of posterolateral decompression with anterior and posterior fusion accomplished with short-segment fixation is a less invasive alternative that 
should not be overlooked when managing this difficult subgroup of patients.

\section{References}

1. Ahlgren BD, Herkowitz HN: A modified posterolateral approach to the thoracic spine. J Spinal Disord 8:69-75, 1995

2. Bhojraj SY, Dandawate AV: Progressive cord compression secondary to thoracic disc lesions in Scheuermann's kyphosis managed by posterolateral decompression, interbody fusion and pedicular fixation. A new approach to management of a rare clinical entity. Eur Spine J 3:66-69, 1994

3. Bhojraj SY, Dandawate AV, Ramakantan R: Preoperative embolisation, transpedicular decompression and posterior stabilisation for metastatic disease of the thoracic spine causing paraplegia. Paraplegia 30:292-299, 1992

4. Burrington JD, Brown C, Wayne ER, et al: Anterior approach to the thoracolumbar spine: technical considerations. Arch Surg 111:456-463, 1976

5. Cook WA: Transthoracic vertebral surgery. Ann Thorac Surg 12:54-68, 1971

6. Hardaker WT, Cook WA Jr, Friedman AH, et al: Bilateral transpedicular decompression and Harrington rod stabilization in the management of severe thoracolumbar burst fractures. Spine 17:162-171, 1992

7. Ikard RW, McCord DH: Thoracoscopic exposure of intervertebral discs. Ann Thorac Surg 61:1267-1268, 1996

8. Maiman DJ, Larson SJ, Luck E, et al: Lateral extracavitary approach to the spine for thoracic disc herniations: report of 23 cases. Neurosurgery 14:178-182, 1984

9. Rosenthal D, Rosenthal R, de Simone A: Removal of a protruded thoracic disc using microsurgical endoscopy. A new technique. Spine 19:1087-1091, 1994

10. Stillerman CB, Chen TC, Diaz Day J, et al: The transfacet pedicle-sparing approach for thoracic disc removal: cadaveric morphometric analysis and preliminary clinical experience. J Neurosurg 83:971-976, 1995

Manuscript received January 16, 1998.

Accepted in final form January 23, 1998.

Address reprint requests to: Kaushik Das, M.D., Department of Neurosurgery, New York Medical College, Munger Pavilion, Valhalla, New York 10595. 